Supporting information for:

\title{
Predicting the denitrification capacity of sandy aquifers from shorter-term incubation experiments and sediment properties
}

W. Eschenbach ${ }^{1,2}$ and R. Well ${ }^{2}$

${ }^{1}$ Soil Science of Temperate Ecosystems, Büsgen-Institute, Büsgenweg 2,

37077 Göttingen, Germany

${ }^{2}$ Johann Heinrich von Thünen-Institut, Federal Research Institute for Rural Areas,

Forestry and Fisheries, Thünen Institute of Climate-Smart Agriculture, Bundesallee 50, 38116 Braunschweig, Germany

Correspondence to: W. Eschenbach (wolfram.eschenbach@ti.bund.de) 


\section{Other possible electron donors}

During incubations Fe and Mn concentrations in the batch solution were always (mostly far) below $1 \mathrm{mg} \mathrm{Fe} \mathrm{L}^{-1}$ and $0,5 \mathrm{mg} \mathrm{Mn} \mathrm{L}^{-1}$. Only some transition zone samples showed $\mathrm{Fe}$ concentrations between 4 and $7 \mathrm{mg} \mathrm{Fe} \mathrm{L}^{-1}$ at the beginning of incubation. The measured concentrations of $\mathrm{Fe}(\mathrm{II})$ and $\mathrm{Mn}(\mathrm{II})$ in the groundwater at the origin of the samples are below $<0.5 \mathrm{mg} \mathrm{Fe} \mathrm{L}^{-1}$ and $<0.1 \mathrm{mg} \mathrm{Mn} \mathrm{L}^{-1}$ in the oxidized zone of both aquifers. Only in the reduced $\mathrm{NO}_{3}{ }^{-}$free zone of both aquifers concentrations of $\mathrm{Fe}(\mathrm{II})$ and $\mathrm{Mn}$ (II) are higher (1 to $7 \mathrm{mg} \mathrm{Fe} \mathrm{L}{ }^{-1}$ and $<0,1 \mathrm{mg} \mathrm{Mn} \mathrm{L}^{-1}$ in the GKA and 4 to $16 \mathrm{mg} \mathrm{Fe} \mathrm{L}^{-1}$ and 0.1 to $1 \mathrm{mg} \mathrm{Mn} \mathrm{L}^{-1}$ in the FFA). Therefore, only solids like e.g. pyrite are possible sources for the electron donors for $\mathrm{NO}_{3}{ }^{-}$reduction in both aquifers and it is assumed that pyrite is the major source for $\mathrm{Fe}(\mathrm{II})$. Recently Korom et al. (2012) indicated that non-pyritic ferrous iron might play a more important role for denitrification than considered up to now. They assume that ferrous iron from amphiboles contributed to denitrification with $2-43 \%$ in a glaciofluvial shallow aquifer in North Dakota.

The $\mathrm{NH}_{4}{ }^{+}$concentrations in the groundwater at the sample origins are below detection limit in the GKA and below $0.5 \mathrm{mg} \mathrm{N} \mathrm{L}^{-1}$ at multilevel well N10 in the FFA, it is assumed that $\mathrm{NH}_{4}{ }^{+}$ is not a significant electron donor during $\mathrm{NO}_{3}{ }^{-}$reduction in both aquifers (see also section 4.5.1 of the manuscript and below).

\section{Limitations of the ${ }^{15} \mathrm{NO}_{3}^{-}$labelling approach}

For the quantification of denitrification ${ }^{15} \mathrm{~N}$ labelled $\mathrm{NO}_{3}{ }^{-}$was used during the conducted anaerobic incubations. ${ }^{15} \mathrm{~N}$ labelling of nitrate can not completely exclude the possible contribution of dissimilatory nitrate reduction to ammonium (DNRA) followed by anaerobic ammonium oxidation (anammox) to the formation of ${ }^{15} \mathrm{~N}$ labelled $\mathrm{N}_{2}$ from the labelled $\mathrm{NO}_{3}{ }^{-}$ during anaerobic incubations.

Under strict anaerobic conditions, DNRA is an alternative pathway for the reduction of $\mathrm{NO}_{3}{ }^{-}$. But DNRA is seldom reported to be the dominant process of $\mathrm{NO}_{3}{ }^{-}$reduction in groundwater systems (Rivett et al., 2008) and chemical modelling by van de Leemput et al. (2011) suggested that DNRA is rather of importance under low $\mathrm{NO}_{3}{ }^{-}$concentrations and high $\mathrm{C}: \mathrm{NO}_{3}^{-}$ratios. But denitrification was presumably not $\mathrm{NO}_{3}^{-}$limited since $\mathrm{NO}_{3}^{-}$ concentrations were always above $1 \mathrm{mg} \mathrm{N} \mathrm{L}^{-1}$ (Wall et al., 2005) during the incubations. DNRA is presumably not an important process during this investigation because the batch 
solutions contained only small amounts of $\mathrm{NH}_{4}{ }^{+}\left(<0,5 \mathrm{mg} \mathrm{N} \mathrm{L}^{-1}\right.$, samples from B2 in depth 8$10 \mathrm{~m} \approx 1 \mathrm{mg} \mathrm{N} \mathrm{L}{ }^{-1}$ ). Also $\mathrm{NH}_{4}{ }^{+}$accumulation was generally not observed during the conducted experiments. Since the incubations were anaerobic $\mathrm{NH}_{4}{ }^{+}$accumulation should be expected if DNRA was a significant contributing process, except anammox consumed the possibly produced $\mathrm{NH}_{4}{ }^{+}$immediately. If significant $\mathrm{N}_{2}$ production via anammox occurred, this would have been difficult to observe since $\mathrm{NH}_{4}{ }^{+}$and $\mathrm{NO}_{2}{ }^{-}$, the educts of this process, came from the same ${ }^{15} \mathrm{~N}$ labelled $\mathrm{NO}_{3}^{-}$pool in the batch solution. (At the beginning of incubation $\mathrm{NO}_{2}^{-}$concentrations were below detection and $\mathrm{NH}_{4}{ }^{+}$concentrations $<0,5 \mathrm{mg} \mathrm{N} \mathrm{L}^{-1}$, respectively.) If anammox contributed significantly to $\mathrm{N}_{2}$ production, than also DNRA must have been a significant process with half the turnover rate of anammox.

Contrary to marine environments, where high rates of anammox are reported (Canfield et al., 2010), in freshwater systems there is not much evidence for anammox (van de Leemput et al., 2011; Burgin and Hamilton, 2007). To our knowledge, there are no studies about anammox in fresh water aquifers, whereas it is reported to exist in wastewater treatment systems, marine sediments and lakes (Jetten et al., 1998;Schubert et al., 2006;Dalsgaard et al., 2005). To distinguish anammox from denitrification during anaerobic incubation experiments ${ }^{15} \mathrm{~N}$ labelled $\mathrm{NO}_{2}{ }^{-}$might be used.

$\mathrm{NH}_{4}{ }^{+}$concentrations in the groundwater are mostly below detection limit in the GKA and in the reduced zone at multilevel well $\mathrm{N} 10$ in the FFA between 0,3 and $0,5 \mathrm{mg} \mathrm{NH}_{4}{ }^{+} \mathrm{L}^{-1}$ (own measurements). Therefore, the possible occurrence of DNRA or DNRA-anammox can not strictly be excluded in both aquifers.

\section{Quantification of total $\mathrm{N}_{2}+\mathrm{N}_{2} \mathrm{O}$ production}

The molecular ion masses 28 and $29\left({ }^{28} \mathrm{~N}_{2},{ }^{29} \mathrm{~N}_{2}\right)$ were recorded for IRMS analysis of denitrification derived ${ }^{15} \mathrm{~N}$ labelled $\mathrm{N}_{2}$ and $\mathrm{N}_{2} \mathrm{O}$. The $\mathrm{N}_{2} \mathrm{O}$ in the headspace samples was reduced to $\mathrm{N}_{2}$ in a reduction column prior to the mass spectrometer entrance. The headspace samples were a mixture of unlabeled $\mathrm{N}_{2}$ und denitrification denitrified ${ }^{15} \mathrm{~N}$ labelled $\mathrm{N}_{2}$ and $\mathrm{N}_{2} \mathrm{O}$. On condition that (i) the ${ }^{15} \mathrm{~N}$ abundance of the denitrified $\mathrm{NO}_{3}{ }^{-}$is known, (ii) denitrification is the sole gaseous nitrogen forming process and (iii) the amount of $\mathrm{N}_{2}$ evolved from the ${ }^{15} \mathrm{~N}$ labelled $\mathrm{NO}_{3}{ }^{-}$pool is small compared with the unlabelled $\mathrm{N}_{2}$ in the sample, the fraction of denitrified $\mathrm{N}_{2}$ in a given mixture can be determined by measuring only ${ }^{29} \mathrm{~N}_{2} /{ }^{28} \mathrm{~N}_{2}$ ratios using the equations provided by Mulvaney (1984) (see also discussion in: (Mulvaney, 1984) and (Eschenbach and Well, 2011)). For the measurement of the ${ }^{15} \mathrm{~N}$ abundance of the 
denitrified $\mathrm{NO}_{3}{ }^{-}$and to check for the conditions mentioned above, replicate samples were measured as described in detail in (Well et al., 1998).

The headspace samples represented a mixture of two binomial $\mathrm{N}_{2}$ isotopologue distributions according to the ${ }^{15} \mathrm{~N}$ abundances of the unlabelled $\mathrm{N}_{2}$ and the ${ }^{15} \mathrm{~N}$ labelled denitrification derived $\left(\mathrm{N}_{2}+\mathrm{N}_{2} \mathrm{O}\right)$, respectively. A high frequency discharge unit was then used for online equilibration of $\mathrm{N}_{2}$ molecules prior to isotope analyses. After equilibration the measured samples consisted of one binomial distribution of $\mathrm{N}_{2}$ isotopologues according to the total ${ }^{15} \mathrm{~N}$ abundance of the mixture. The ${ }^{15} \mathrm{~N}$ abundance of the denitrified $\mathrm{NO}_{3}{ }^{-}$can then be calculated from the measurement of the ${ }^{29} \mathrm{~N}_{2} /{ }^{28} \mathrm{~N}_{2}$ ratios of unequilibrated and equilibrated replicate samples (Well et al., 1998).

\section{Fit between $\mathrm{NO}_{3}{ }^{-}$consumption and $\left(\mathrm{N}_{2}+\mathrm{N}_{2} \mathrm{O}\right)$ production}

The $\mathrm{NO}_{3}{ }^{-}$decrease during incubations showed the same pattern as the measured production of $\left(\mathrm{N}_{2}+\mathrm{N}_{2} \mathrm{O}\right)$ by GC-IRMS. The measurement of $\left(\mathrm{N}_{2}+\mathrm{N}_{2} \mathrm{O}\right)$ production by GC-IRMS was more precise and had a lower detection limit compared to the measurement of $\mathrm{NO}_{3}{ }^{-}$consumption (compare Fig. 1a and Fig. S3a).

The $\mathrm{N}$ balance between the $\mathrm{NO}_{3}{ }^{-}$content at the start of incubations and the sum of $\mathrm{NO}_{3}{ }^{-}$and denitrification derived $\left(\mathrm{N}_{2}+\mathrm{N}_{2} \mathrm{O}\right)$ concentrations during incubation was for most of the incubated samples $<1 \mathrm{mg} \mathrm{N} /$ batch assay. The samples with the highest measured production of $\left(\mathrm{N}_{2}+\mathrm{N}_{2} \mathrm{O}\right)$ showed also the highest deviation between the amount of $\mathrm{NO}_{3}{ }^{-}$consumed and the measured production of $\left(\mathrm{N}_{2}+\mathrm{N}_{2} \mathrm{O}\right)$ (compare Fig. 1c and Fig. S3c).

\section{Recommendations for future anaerobic incubations}

\section{Control of air contamination during incubation experiments}

Canfield et al. (2010) recommended to de-aerate rubber septa by boiling them for 24 hour in water and store them in a He atmosphere before use. An elegant way to check for possible air contamination is the measurement of Ar in the headspace of the transfusion bottles during incubation. Increasing Ar concentrations are an indicator of air contaminations during incubation. Unfortunately we were not able to measure Ar during the incubations, due to instrumental restrictions. 
Table S1. Sediment parameters and basic properties of all incubated samples

\begin{tabular}{|c|c|c|c|c|c|c|c|c|c|c|c|}
\hline \multirow{2}{*}{$\begin{array}{l}\text { Sample } \\
\text { location }\end{array}$} & \multirow{2}{*}{$\begin{array}{c}\text { Depth } \\
\text { interval } \\
{[\mathrm{m}]}\end{array}$} & \multirow[t]{2}{*}{$\mathrm{SG}^{\mathrm{a}}$} & \multirow{2}{*}{$\begin{array}{c}\mathrm{SO}_{4}{ }^{2-\mathrm{b}} \\
\mathrm{mg} \mathrm{S} \mathrm{kg}^{-1}\end{array}$} & $\mathrm{DOC}^{\mathrm{c}}$ & $\mathrm{C}_{\mathrm{hws}}{ }^{\mathrm{d}}$ & $\mathrm{C}_{\mathrm{l}}^{\mathrm{e}}$ & $\mathrm{C}_{\text {org }}$ & \multirow{2}{*}{$\begin{array}{l}\text { total-S } \\
\mathrm{mg} \mathrm{S} \\
\mathrm{kg}^{-1}\end{array}$} & \multirow{2}{*}{$\begin{array}{l}\text { total-N } \\
\mathrm{mg} \mathrm{N} \\
\mathrm{kg}^{-1}\end{array}$} & \multirow{2}{*}{$\begin{array}{r}\text { Sand } \\
\\
{[\%}\end{array}$} & \multirow[t]{2}{*}{ Silt } \\
\hline & & & & \multicolumn{4}{|c|}{$\mathrm{mg} \mathrm{C} \mathrm{kg}$} & & & & \\
\hline FFA B1 & $6.0-7.0$ & $s^{n}$ & 3.3 & 7.2 & 30.3 & 82.2 & 643 & 86 & 33 & 95.0 & 5.0 \\
\hline FFA B1 & $7.0-8.0$ & $\mathrm{~s}$ & 3.3 & 5.7 & 32.3 & 887.0 & 5955 & 603 & 94 & 94.8 & 5.2 \\
\hline FFA B2 & $2.0-3.0$ & $\mathrm{~ns}^{\mathrm{n}}$ & 10.2 & 11.5 & 20.0 & 2.7 & 237 & 29 & 26 & 98.9 & 0.2 \\
\hline FFA B2 & $3.0-4.0$ & $\mathrm{n} \mathrm{s}^{\mathrm{n}}$ & 25.3 & 10.2 & 17.2 & 2.7 & 203 & 38 & 23 & 98.9 & 0.2 \\
\hline FFA B2 & $4.0-5.0$ & $\mathrm{n} \mathrm{s}^{\mathrm{n}}$ & 19.5 & 8.9 & 21.6 & 228.6 & 545 & 46 & 54 & 96.4 & 1.3 \\
\hline FFA B2 & $8.0-9.0$ & $s^{n}$ & 0.0 & 6.9 & 33.8 & 93.9 & 1625 & 176 & 31 & 40.4 & 59.6 \\
\hline FFA B2 & $9.0-10.0$ & $s^{n}$ & 0.9 & 6.2 & 40.0 & 116.9 & 538 & 156 & 28 & 94.7 & 5.3 \\
\hline FFA B4 & $7.0-8.0$ & $\mathrm{~s}$ & n.d. ${ }^{1}$ & n.d. ${ }^{1}$ & n.d. ${ }^{1}$ & n.d. ${ }^{1}$ & 483 & 220 & 21 & 97.3 & 2.7 \\
\hline FFA B4 & $8.0-9.0$ & $\mathrm{~s}$ & n.d. ${ }^{1}$ & n.d. ${ }^{1}$ & n.d. ${ }^{1}$ & n.d. ${ }^{1}$ & 1114 & 359 & 39 & 95.4 & 4.7 \\
\hline FFA B6 & $2.0-3.0$ & $\mathrm{n} \mathrm{s}^{\mathrm{n}}$ & 17.7 & 11.6 & 22.1 & 259.6 & 695 & 56 & 41 & 97.8 & 0.6 \\
\hline FFA B6 & $3.0-4.0$ & $\mathrm{n} \mathrm{s}^{\mathrm{n}}$ & 23.3 & 10.3 & 21.6 & 172.5 & 1047 & 59 & 46 & 97.8 & 0.4 \\
\hline FFA N10 & $4.5-5.0$ & $s^{n}$ & 5.4 & 9.2 & 22.2 & 462.7 & 1291 & 50 & 87 & 94.9 & 1.0 \\
\hline FFA N10 & $5.0-5.5$ & $s^{n}$ & 3.8 & 9.6 & 27.6 & 206.9 & 737 & 49 & 55 & 98.0 & 0.3 \\
\hline FFA N10 & $5.5-6.0$ & $s^{n}$ & 12.8 & 10.8 & 28.4 & 160.6 & 687 & 49 & 36 & 97.4 & 0.4 \\
\hline FFA N10 & $7.7-8.3$ & $s^{n}$ & n.d. ${ }^{1}$ & n.d. ${ }^{1}$ & 41.2 & n.d. ${ }^{1}$ & 311 & 57 & 10 & 96.3 & 3.8 \\
\hline FFA N10 & $8.3-8.6$ & $s^{n}$ & n.d. ${ }^{1}$ & n.d. ${ }^{1}$ & 42.5 & n.d. ${ }^{1}$ & 320 & 47 & 11 & 97.9 & 2.2 \\
\hline FFA N10 & $10.0-10.4$ & $\mathrm{~S}$ & n.d. ${ }^{1}$ & n.d. ${ }^{1}$ & n.d. ${ }^{1}$ & n.d. ${ }^{1}$ & 310 & 45 & 18 & 96.3 & 3.7 \\
\hline FFA N10 & $10.4-10.7$ & $\mathrm{~s}$ & n.d. ${ }^{1}$ & n.d. ${ }^{1}$ & n.d. ${ }^{1}$ & n.d. ${ }^{1}$ & 5627 & 464 & 113 & 96.4 & 3.6 \\
\hline FFA N10 & $12.0-13.0$ & $\mathrm{~s}$ & n.d. ${ }^{1}$ & n.d. ${ }^{1}$ & 0.0 & n.d. ${ }^{1}$ & 2554 & 558 & 64 & 96.7 & 3.3 \\
\hline FFA N10 & $13.0-14.0$ & $\mathrm{~s}$ & n.d. ${ }^{1}$ & n.d. ${ }^{1}$ & 39.7 & n.d. ${ }^{1}$ & 1848 & 588 & 53 & 95.1 & 4.9 \\
\hline FFA N10 & $16.0-17.0$ & $\mathrm{~s}$ & 1.1 & 5.7 & 42.6 & 241.0 & 2608 & 448 & 51 & 97.2 & 2.8 \\
\hline FFA N10 & $17.0-18.0$ & $\mathrm{~S}$ & n.d. ${ }^{1}$ & n.d. ${ }^{1}$ & 41.1 & n.d. ${ }^{1}$ & 2504 & 441 & 48 & 96.9 & 3.1 \\
\hline GKA & $8.0-9.0$ & $\mathrm{~ns}^{\mathrm{n}}$ & 14.5 & 8.1 & 18.3 & 1.8 & 102 & 54 & 9 & 96.8 & 1.4 \\
\hline GKA & $9.0-10.0$ & $\mathrm{~ns}^{\mathrm{n}}$ & 14.5 & 9.0 & 14.9 & 0.9 & 76 & 38 & 6 & 97.3 & 0.9 \\
\hline GKA & $22.0-23.0$ & $\mathrm{~ns}^{\mathrm{n}}$ & 11.1 & 8.6 & 43.8 & 221.3 & 176 & 42 & 15 & 95.4 & 1.2 \\
\hline GKA & $23.0-24.0$ & $\mathrm{~ns}^{\mathrm{n}}$ & 10.8 & 9.4 & 33.7 & 50.3 & 192 & 36 & 23 & 96.0 & 0.9 \\
\hline GKA & $25.9-27.0$ & $\mathrm{~s}$ & 8.2 & 6.1 & 31.1 & 1021.2 & 2553 & 682 & 69 & 87.6 & 12.4 \\
\hline GKA & $27.0-28.3$ & $\mathrm{~s}$ & 4.8 & 5.8 & 39.0 & 1531.1 & 6373 & 989 & 127 & 79.6 & 20.4 \\
\hline GKA & $28.3-29.3$ & $\mathrm{~s}$ & 10.3 & 8.1 & 27.4 & 2504.9 & 4159 & 883 & 114 & 76.8 & 21.3 \\
\hline GKA & $29.3-30.3$ & $\mathrm{~s}$ & 12.7 & 6.6 & 26.2 & 2205.8 & 4543 & 760 & 96 & 83.9 & 14.2 \\
\hline GKA & $30.3-31.2$ & $\mathrm{~s}$ & 13.6 & 5.2 & 28.9 & 347.7 & 784 & 509 & 14 & 97.6 & 2.2 \\
\hline GKA & $31.3-32.0$ & $\mathrm{~s}$ & 18.1 & 9.9 & 42.6 & 192.0 & 834 & 494 & 27 & 96.5 & 3.2 \\
\hline GKA & $32.9-33.7$ & $\mathrm{~s}$ & 20.2 & 5.1 & 20.8 & 377.4 & 821 & 630 & 23 & 96.9 & 2.8 \\
\hline GKA & $33.7-34.7$ & $\mathrm{~s}$ & 15.6 & 5.3 & 29.2 & 150.5 & 752 & 510 & 17 & 98.5 & 1.4 \\
\hline GKA & $35.7-36.7$ & $\mathrm{~s}$ & 2.2 & 5.4 & 32.0 & 2391.1 & 8972 & 708 & 120 & 96.9 & 3.1 \\
\hline GKA & $36.7-37.7$ & $\mathrm{~s}$ & 5.1 & 5.5 & 22.4 & 37.7 & 232 & 677 & 3 & 98.8 & 1.2 \\
\hline GKA & $37.7-38.7$ & $\mathrm{~s}$ & 0.5 & 4.7 & 23.2 & 447.4 & 1162 & 379 & 30 & 97.8 & 2.3 \\
\hline GKA & $65.1-65.4$ & $\mathrm{~s}$ & 1.8 & 6.2 & 23.7 & 239.8 & 1009 & 716 & 39 & 89.4 & 10.7 \\
\hline GKA & $67.1-67.5$ & $\mathrm{~ns}$ & 0.3 & 6.9 & 56.5 & 132.1 & 358 & 196 & 21 & 92.1 & 7.9 \\
\hline GKA & $67.5-68.0$ & $\mathrm{~ns}$ & 3.5 & 5.2 & 58.5 & n.d. ${ }^{1}$ & 377 & 194 & 44 & 94.7 & 5.3 \\
\hline
\end{tabular}

\footnotetext{
${ }^{\mathrm{a}}$ sediment group; ${ }^{\mathrm{b}}$ extractable sulfate-S; ${ }^{\mathrm{c}}$ extractable dissolved organic carbon; ${ }^{\mathrm{d}}$ extractable hot-water soluble carbon; ${ }^{\mathrm{e}} \mathrm{KMnO}_{4}$ labile organic carbon; ${ }^{1}$ n.d.: not determined; $\mathrm{n}$ s non-sulphidic; s sulphidic aquifer material, $\mathrm{n} \mathrm{s}$ and $\mathrm{s}$ with the subscript $\mathrm{n}$ indicates $\mathrm{NO}_{3}{ }^{-}$-bearing samples.
} 
Table S2. Denitrification rates, long-term denitrification capacity, stock of reduced compounds, sulphate formation capacity and estimated minimal lifetime of denitrification of all incubated samples.

\begin{tabular}{|c|c|c|c|c|c|c|c|c|c|c|}
\hline $\begin{array}{l}\text { Sample } \\
\text { location }\end{array}$ & $\begin{array}{l}\text { Depth } \\
\text { interval }\end{array}$ & $\mathrm{SG}^{\mathrm{a}}$ & $\mathrm{D}_{\mathrm{r}}(7)^{\mathrm{b}}$ & $\begin{array}{c}\mathrm{D}_{\text {cum }} \\
(365) \\
\mathrm{d}\end{array}$ & $\mathrm{SRC}^{\mathrm{e}}$ & $\mathrm{SRC}_{\mathrm{C}}{ }^{\mathrm{f}}$ & $\mathrm{SRC}_{\mathrm{S}}{ }^{\mathrm{g}}$ & $\mathrm{aF}_{\mathrm{SRC}}{ }^{\mathrm{h}}$ & $\mathrm{SFC}^{\mathrm{i}}$ & $\begin{array}{c}\mathrm{em} \\
\mathrm{LoD}^{\mathrm{j}}\end{array}$ \\
\hline & {$[\mathrm{m}]$} & & $\begin{array}{c}\mu \mathrm{g} \mathrm{N} \\
\mathrm{kg}^{-1} \\
\mathrm{~d}^{-1}\end{array}$ & $\begin{array}{l}\mathrm{mg} \mathrm{N} \\
\mathrm{kg}^{-1} \\
\mathrm{yr}^{-1}\end{array}$ & & $\mathrm{~g} \mathrm{~N} \mathrm{~kg}^{-1}$ & & $\% \mathrm{yr}^{-1}$ & $\begin{array}{c}\mathrm{mg} \mathrm{S} \\
\mathrm{kg}^{-1} \mathrm{yr}^{-1}\end{array}$ & yr \\
\hline FFA B1 & $6.0-7.0$ & $s^{n}$ & 51.66 & 17.18 & 659.6 & 599.5 & 60.1 & 2.60 & 6.1 & 5.0 \\
\hline FFA B1 & $7.0-8.0$ & $\mathrm{~s}$ & 33.89 & 56.24 & 5974.2 & 5552.7 & 421.5 & 0.94 & 39.4 & 44.8 \\
\hline FFA B2 & $2.0-3.0$ & $\mathrm{~ns}^{\mathrm{n}}$ & 1.27 & 0.19 & 240.8 & 220.7 & 20.1 & 0.08 & 0.1 & 1.8 \\
\hline FFA B2 & $3.0-4.0$ & $\mathrm{~ns}^{\mathrm{n}}$ & 2.12 & 0.37 & 215.4 & 189.2 & 26.3 & 0.17 & -0.1 & 1.6 \\
\hline FFA B2 & $4.0-5.0$ & $\mathrm{n} \mathrm{s}^{\mathrm{n}}$ & 35.27 & 4.34 & 540.2 & 508.0 & 32.2 & 0.80 & 1.0 & 4.1 \\
\hline FFA B2 & $8.0-9.0$ & $s^{n}$ & 21.05 & 10.53 & 1638.2 & 1515.5 & 122.7 & $0.64^{(10.0)}$ & 3.5 & 12.3 \\
\hline FFA B2 & $9.0-10.0$ & $s^{n}$ & 41.41 & 12.68 & 610.7 & 502.0 & 108.7 & $2.08^{(26.4)}$ & 2.2 & 4.6 \\
\hline FFA B4 & $7.0-8.0$ & $\mathrm{~s}$ & 45.67 & 20.16 & 603.6 & 450.2 & 153.4 & 3.34 & 9.6 & 4.5 \\
\hline FFA B4 & $8.0-9.0$ & $\mathrm{~s}$ & 25.24 & 34.09 & 1289.5 & 1038.9 & 250.7 & 2.64 & 22.0 & 9.7 \\
\hline FFA B6 & $2.0-3.0$ & $\mathrm{~ns}^{\mathrm{n}}$ & 11.53 & 2.64 & 687.0 & 648.9 & 39.1 & 0.38 & 0.3 & 5.2 \\
\hline FFA B6 & $3.0-4.0$ & $n s^{n}$ & 6.93 & 1.46 & 1017.4 & 976.5 & 40.9 & 0.14 & 0.1 & 7.6 \\
\hline FFA N10 & $4.5-5.0$ & $s^{n}$ & 35.97 & 8.69 & 1239.0 & 1204.1 & 34.8 & 0.70 & 1.5 & 9.3 \\
\hline FFA N10 & $5.0-5.5$ & $s^{n}$ & 61.03 & 8.75 & 721.6 & 687.1 & 34.5 & 1.21 & 2.1 & 5.4 \\
\hline FFA N10 & $5.5-6.0$ & $s^{n}$ & 36.99 & 7.82 & 674.6 & 640.3 & 34.3 & 1.16 & 5.2 & 5.1 \\
\hline FFA N10 & $7.7-8.3$ & $s^{n}$ & 33.71 & 15.04 & 329.5 & 290.0 & 39.5 & 4.56 & 1.5 & 2.5 \\
\hline FFA N10 & $8.3-8.6$ & $s^{n}$ & 20.25 & 15.17 & 331.5 & 298.7 & 32.9 & 4.58 & 6.9 & 2.5 \\
\hline FFA N10 & $10.0-10.4$ & s & 12.34 & 17.45 & 320.6 & 289.3 & 31.3 & 5.44 & 5.4 & 2.4 \\
\hline FFA N10 & $10.4-10.7$ & s & 23.75 & 50.07 & 5571.6 & 5247.7 & 323.9 & 0.90 & 9.4 & 41.8 \\
\hline FFA N10 & $12.0-13.0$ & s & 26.47 & 52.84 & 2771.3 & 2381.7 & 389.6 & 1.91 & 37.9 & 20.8 \\
\hline FFA N10 & $13.0-14.0$ & s & 35.58 & 38.04 & 2134.1 & 1723.3 & 410.8 & 1.78 & 18.2 & 16.0 \\
\hline FFA N10 & $16.0-17.0$ & s & 69.90 & 46.65 & 2744.7 & 2431.5 & 313.2 & $1.70^{(6.3)}$ & 23.6 & 20.6 \\
\hline FFA N10 & $17.0-18.0$ & $\mathrm{~s}$ & 34.48 & 46.55 & 2642.7 & 2335.0 & 307.8 & $1.76^{(6.3)}$ & 36.8 & 19.8 \\
\hline GKA & $8.0-9.0$ & $n s^{n}$ & 0.81 & 0.63 & 132.6 & 95.0 & 37.6 & 0.47 & 0.9 & 1.0 \\
\hline GKA & $9.0-10.0$ & $\mathrm{~ns}^{\mathrm{n}}$ & 0.71 & 0.34 & 97.1 & 70.7 & 26.4 & 0.35 & 0.4 & 0.7 \\
\hline GKA & $22.0-23.0$ & $\mathrm{n} \mathrm{s}^{\mathrm{n}}$ & 14.68 & 1.57 & 193.3 & 164.2 & 29.1 & 0.81 & 0.2 & 1.5 \\
\hline GKA & $23.0-24.0$ & $\mathrm{~ns}^{\mathrm{n}}$ & 31.77 & 2.83 & 204.5 & 179.2 & 25.3 & 1.38 & 0.0 & 1.5 \\
\hline GKA & $25.9-27.0$ & s & 26.36 & 15.63 & 2857.4 & 2381.0 & 476.4 & 0.55 & 1.2 & 21.4 \\
\hline GKA & $27.0-28.3$ & $\mathrm{~s}$ & 29.43 & 41.82 & 6634.0 & 5943.2 & 690.8 & $0.63^{(4.9)}$ & 8.3 & 49.8 \\
\hline GKA & $28.3-29.3$ & s & 46.38 & 37.82 & 4495.6 & 3878.5 & 617.2 & $0.84^{(7.3)}$ & 13.8 & 33.7 \\
\hline GKA & $29.3-30.3$ & s & 57.08 & 35.49 & 4766.8 & 4236.0 & 530.8 & $0.74^{(6.4)}$ & 8.1 & 35.8 \\
\hline GKA & $30.3-31.2$ & s & 26.07 & 6.54 & 1086.9 & 731.4 & 355.4 & 0.60 & 3.8 & 8.2 \\
\hline GKA & $31.3-32.0$ & s & 14.06 & 4.09 & 1122.4 & 777.7 & 344.7 & 0.36 & 5.0 & 8.4 \\
\hline GKA & $32.9-33.7$ & s & 38.39 & 7.28 & 1206.0 & 765.6 & 440.4 & 0.60 & 10.2 & 9.1 \\
\hline GKA & $33.7-34.7$ & $\mathrm{~s}$ & 62.14 & 12.25 & 1057.4 & 700.9 & 356.6 & 1.16 & 17.7 & 7.9 \\
\hline GKA & $35.7-36.7$ & $\mathrm{~s}$ & 64.30 & 52.46 & 8861.3 & 8366.7 & 494.6 & $0.59^{(4.6)}$ & 30.0 & 66.5 \\
\hline GKA & $36.7-37.7$ & $\mathrm{~s}$ & 87.51 & 11.07 & 689.6 & 216.7 & 472.8 & 1.60 & 9.2 & 5.2 \\
\hline GKA & $37.7-38.7$ & s & 109.2 & 12.06 & 1347.7 & 1083.1 & 264.7 & $0.89^{(15.3)}$ & 4.6 & 10.1 \\
\hline GKA & $65.1-65.4$ & s & 33.12 & 13.22 & 1441.2 & 941.3 & 499.9 & 0.92 & 1.3 & 10.8 \\
\hline GKA & $67.1-67.5$ & $\mathrm{n} \mathrm{s}$ & 30.54 & 8.18 & 471.0 & 333.8 & 137.2 & 1.74 & 1.3 & 3.5 \\
\hline GKA & $67.5-68.0$ & $\mathrm{n} \mathrm{s}$ & 23.62 & 8.11 & 487.1 & 351.5 & 135.6 & 1.67 & 0.7 & 3.7 \\
\hline
\end{tabular}


${ }^{\mathrm{a}}$ sediment group; ${ }^{\mathrm{b}}$ initial denitrification rate; ${ }^{\mathrm{c}}$ average denitrification rate after one year; ${ }^{\mathrm{d}}$ cumulative denitrification after one year; ${ }^{\mathrm{e}}$ depot of reactive compounds (SRC); ${ }^{\mathrm{f}}$ concentration of reduced compounds derived from measured $\mathrm{C}_{\mathrm{org}},{ }^{\mathrm{g}}$ concentration of reduced compounds derived from total-S values; ${ }^{\mathrm{h}}$ fraction of SRC available for denitrification during one year of incubation, in parenthesis $\mathrm{aF}_{\mathrm{SRC}}$ from the intensive treatment; ${ }^{\mathrm{i}}$ sulphate formation capacity (SFC); ${ }^{\mathrm{j}}$ estimated minimal lifetime of denitrification; $\mathrm{n} s$ non-sulphidic; $\mathrm{s}$ sulphidic aquifer material, $\mathrm{n} \mathrm{s}$ and $\mathrm{s}$ with the subscript $\mathrm{n}$ indicates $\mathrm{NO}_{3}{ }^{-}$-bearing samples.

Table S3. Simple regression between $D_{\text {cum }}(365)$ and sediment parameters $(X)$,

$f^{B-C}\left(D_{\text {cum }}(365)\right)=\mathrm{A}+\mathrm{B} \times f^{B-C}(X)$. Regressions with $\mathrm{C}_{\text {org }}$, total-S are listed for each partial data set. Regression with a third independent sediment variable are only given, if correlation coefficient were better compared to correlations with $\mathrm{C}_{\text {org }}$ or total-S.

\begin{tabular}{|c|c|c|c|c|c|}
\hline Data set & $\mathrm{X}^{\mathrm{a}}$ & $\mathrm{N}^{\mathrm{b}}$ & $\mathrm{R}^{\mathrm{c}}$ & A & B \\
\hline Whole data set & $\mathrm{C}_{\text {org }}$ & 151 & 0.80 & -11.022 & 2.654 \\
\hline whole data set & total-S & 151 & 0.71 & -2.397 & 0.805 \\
\hline whole data set & $\mathrm{C}_{\mathrm{l}}$ & 111 & 0.83 & -1.028 & 0.492 \\
\hline FFA & $\mathrm{C}_{\text {org }}$ & 86 & 0.72 & -26.950 & 8.017 \\
\hline FFA & total-S & 86 & 0.83 & -14.879 & 6.312 \\
\hline FFA & DOC $_{\text {extr }}$ & 46 & 0.84 & 10.503 & -0.495 \\
\hline GKA & $\mathrm{C}_{\text {org }}$ & 65 & 0.93 & -9.525 & 2.457 \\
\hline GKA & total-S & 65 & 0.86 & -0.252 & 0.026 \\
\hline GKA & $\mathrm{C}_{1}$ & 65 & 0.93 & -0.730 & 0.416 \\
\hline non-sulphidic & $\mathrm{C}_{\text {org }}$ & 44 & 0.52 & -5.434 & 1.205 \\
\hline non-sulphidic & total-S & 44 & 0.77 & -231.440 & 284.854 \\
\hline non-sulphidic & $\mathrm{C}_{\mathrm{hws}}$ & 44 & 0.77 & -164.600 & 233.898 \\
\hline sulphidic & $\mathrm{C}_{\text {org }}$ & 107 & 0.66 & -3.097 & 1.293 \\
\hline sulphidic & total-S & 107 & 0.40 & 2.747 & 0.001 \\
\hline sulphidic & $\mathrm{C}_{1}$ & 67 & 0.60 & -0.119 & 0.638 \\
\hline $\mathrm{NO}_{3}{ }^{-}$-bearing & $\mathrm{C}_{\text {org }}$ & 64 & 0.58 & -4.946 & 0.661 \\
\hline $\mathrm{NO}_{3}{ }^{-}$-bearing & total-S & 64 & 0.67 & -268.670 & 312.977 \\
\hline $\mathrm{NO}_{3}{ }^{-}$-bearing & $\mathrm{C}_{1}$ & 56 & 0.73 & -0.737 & 0.267 \\
\hline $\mathrm{NO}_{3}{ }^{-}$-free & $\mathrm{C}_{\text {org }}$ & 87 & 0.77 & -5.862 & 1.623 \\
\hline $\mathrm{NO}_{3}{ }^{-}$-free & total-S & 87 & 0.32 & 3.741 & 0.004 \\
\hline transition zone & $\mathrm{C}_{\text {org }}$ & 28 & 0.58 & 18.117 & -4.020 \\
\hline transition zone & total-S & 28 & 0.20 & -178.180 & 277.350 \\
\hline transition zone & $\mathrm{C}_{1}$ & 20 & 0.73 & 192.880 & -190.340 \\
\hline
\end{tabular}

${ }^{\mathrm{a}}$ Independent sediment parameter

${ }^{\mathrm{b}}$ Sample number

${ }^{\mathrm{c}}$ Correlation coefficient 
Table: S4. Ratios of modelled $D_{\text {cum }}(365)$ vs measured $D_{\text {cum }}(365)$ (group means with standard deviation, ranges in parentheses) for samples with high $\left(>20 \mathrm{mg} \mathrm{N} \mathrm{kg}^{-1}\right)$ and low $D_{\text {cum }}(365)(<20 \mathrm{mg}$ $\left.\mathrm{N} \mathrm{kg}^{-1}\right)$.

\begin{tabular}{|c|c|c|c|c|c|c|}
\hline \multirow{3}{*}{ Data set } & \multicolumn{6}{|c|}{ Modelled $\mathrm{D}_{\text {cum }}(365) /$ Measured $\mathrm{D}_{\text {cum }}(365)$} \\
\hline & \multicolumn{3}{|c|}{ Multiple regressions } & \multicolumn{3}{|c|}{ Simple regressions } \\
\hline & Selection $\mathrm{I}^{\mathrm{a}}$ & Selection II $^{\mathrm{b}}$ & Selection III ${ }^{\mathrm{c}}$ & $\mathrm{C}_{\text {org }}$ & Total-S & Best $^{\mathrm{d}}$ \\
\hline & \multicolumn{6}{|c|}{$\mathrm{D}_{\text {cum }}(365) \geq 20 \mathrm{mg} \mathrm{N} \mathrm{kg}^{-1} \mathrm{yr}^{-1}$} \\
\hline Whole data set & $\begin{array}{l}0.88 \pm 0.33 \\
(0.33-1.67)\end{array}$ & $\begin{array}{l}0.89 \pm 0.28 \\
(0.39-1.26)\end{array}$ & $\begin{array}{l}0.87 \pm 0.24 \\
(0.55-1.30)\end{array}$ & $\begin{array}{l}0.86 \pm 0.32 \\
(0.29-1.53)\end{array}$ & $\begin{array}{l}0.68 \pm 0.25 \\
(0.42-1.54)\end{array}$ & $\begin{array}{l}0.83 \pm 0.38 \\
(0.22-1.35)\end{array}$ \\
\hline FFA & $\begin{array}{l}0.86 \pm 0.12 \\
(0.71-1.26)\end{array}$ & $\begin{array}{l}0.86 \pm 0.50 \\
(0.79-0.93)\end{array}$ & $\begin{array}{l}0.84 \pm 0.07 \\
(0.74-0.94)\end{array}$ & $\begin{array}{l}0.71 \pm 0.17 \\
(0.30-1.08)\end{array}$ & $\begin{array}{l}0.86 \pm 0.15 \\
(0.68-1.29)\end{array}$ & $\begin{array}{l}0.57 \pm 0.06 \\
(0.49-0.66)\end{array}$ \\
\hline GKA & $\begin{array}{l}0.89 \pm 0.33 \\
(0.41-1.47)\end{array}$ & $\begin{array}{l}1.14 \pm 0.18 \\
(0.78-1.38)\end{array}$ & $\begin{array}{l}1.08 \pm 0.19 \\
(0.79-1.34)\end{array}$ & $\begin{array}{l}1.14 \pm 0.19 \\
(0.88-1.46)\end{array}$ & $\begin{array}{l}0.84 \pm 0.30 \\
(0.39-1.38)\end{array}$ & $\begin{array}{l}1.13 \pm 0.26 \\
(0.67-1.51)\end{array}$ \\
\hline \multirow[t]{2}{*}{ sulphidic } & $\begin{array}{l}0.73 \pm 0.22 \\
(0.44-1.35) \\
\end{array}$ & $\begin{array}{l}0.78 \pm 0.16 \\
(0.57-1.13) \\
\end{array}$ & $\begin{array}{l}1.15 \pm 0.38 \\
(0.81-2.05) \\
\end{array}$ & $\begin{array}{l}0.74 \pm 0.22 \\
(0.43-1.36)\end{array}$ & $\begin{array}{l}0.33 \pm 0.09 \\
(0.23-0.68)\end{array}$ & $\begin{array}{l}0.66 \pm 0.25 \\
(0.28-1.19) \\
\end{array}$ \\
\hline & \multicolumn{6}{|c|}{$\mathrm{D}_{\text {cum }}(365)<20 \mathrm{mg} \mathrm{N} \mathrm{kg}^{-1} \mathrm{yr}^{-1}$} \\
\hline Whole data set & $\begin{array}{l}2.29 \pm 3.06 \\
(0.20-18.28)\end{array}$ & $\begin{array}{l}1.90 \pm 2.27 \\
(0.17-11.08)\end{array}$ & $\begin{array}{l}1.38 \pm 1.02 \\
(0.34-6.23)\end{array}$ & $\begin{array}{l}2.69 \pm 4.40 \\
(0.23-26.07)\end{array}$ & $\begin{array}{l}3.03 \pm 3.85 \\
(0.20-18.32)\end{array}$ & $\begin{array}{l}1.72 \pm 1.49 \\
(0.23-8.79)\end{array}$ \\
\hline FFA & $\begin{array}{l}2.52 \pm 3.03 \\
(0.23-12.41)\end{array}$ & $\begin{array}{l}1.77 \pm 1.44 \\
(0.34-5.69)\end{array}$ & $\begin{array}{l}1.14 \pm 0.66 \\
(0.26-3.41)\end{array}$ & $\begin{array}{l}3.56 \pm 4.90 \\
(0.24-20.27)\end{array}$ & $\begin{array}{l}2.63 \pm 3.39 \\
(0.25-13.64)\end{array}$ & $\begin{array}{l}2.19 \pm 2.53 \\
(0.18-11.82)\end{array}$ \\
\hline GKA & $\begin{array}{l}1.73 \pm 1.29 \\
(0.31-5.51)\end{array}$ & $\begin{array}{l}1.35 \pm 0.71 \\
(0.23-3.10)\end{array}$ & $\begin{array}{l}1.19 \pm 0.43 \\
(0.30-2.16)\end{array}$ & $\begin{array}{l}1.39 \pm 0.82 \\
(0.23-3.99)\end{array}$ & $\begin{array}{l}1.76 \pm 1.38 \\
(0.34-6.02)\end{array}$ & $\begin{array}{l}1.35 \pm 0.68 \\
(0.23-3.02)\end{array}$ \\
\hline non-sulphidic & $\begin{array}{l}1.36 \pm 1.04 \\
(0.18-5.23)\end{array}$ & $\begin{array}{l}1.36 \pm 1.04 \\
(0.18-5.23)\end{array}$ & $\begin{array}{l}1.09 \pm 0.45 \\
(0.52-0.45)\end{array}$ & $\begin{array}{l}1.94 \pm 2.39 \\
(0.21-10.45)\end{array}$ & $\begin{array}{l}1.47 \pm 1.00 \\
(0.18-8.25)\end{array}$ & $\begin{array}{l}1.55 \pm 0.94 \\
(0.24-7.26)\end{array}$ \\
\hline sulphidic & $\begin{array}{l}1.49 \pm 0.84 \\
(0.51-4.33)\end{array}$ & $\begin{array}{l}1.29 \pm 0.66 \\
(0.33-3.13)\end{array}$ & $\begin{array}{l}1.39 \pm 0.60 \\
(0.43-3.19)\end{array}$ & $\begin{array}{l}1.48 \pm 0.84 \\
(0.50-4.36)\end{array}$ & $\begin{array}{l}1.27 \pm 0.61 \\
(0.69-3.69)\end{array}$ & $\begin{array}{l}1.46 \pm 0.76 \\
(0.44-3.49)\end{array}$ \\
\hline transition zone & $\begin{array}{l}1.03 \pm 0.22 \\
(0.71-1.52)\end{array}$ & $\begin{array}{l}1.03 \pm 0.22 \\
(0.67-1.56)\end{array}$ & $\begin{array}{l}1.01 \pm 0.13 \\
(0.84-1.27)\end{array}$ & $\begin{array}{l}1.05 \pm 0.27 \\
(0.64-1.77)\end{array}$ & $\begin{array}{l}1.07 \pm 0.32 \\
(0.67-1.73)\end{array}$ & $\begin{array}{l}1.03 \pm 0.24 \\
(0.72-1.58)\end{array}$ \\
\hline
\end{tabular}

${ }^{\mathrm{a}} \mathrm{C}_{\mathrm{org}}$ and total-S;

${ }^{\mathrm{b}}$ two sediment parameters giving highest correlation coefficient;

c stepwise multiple regression;

${ }^{\mathrm{d}}$ simple regression with the sediment parameter giving the best correlations with $D_{\text {cum }}(365)$;

Table S5. Lambda values of the Box-Cox transformed sediment parameters

\begin{tabular}{|c|c|c|c|c|c|c|c|c|c|c|c|c|}
\hline \multirow[t]{2}{*}{ Data set } & \multicolumn{12}{|c|}{ Lambda values } \\
\hline & $\mathrm{D}_{\mathrm{r}}(7)$ & $\mathrm{D}_{\mathrm{r}}(84)$ & $\mathrm{D}_{\mathrm{r}}(168)$ & $\begin{array}{l}\mathrm{D}_{\text {cum }} \\
(365)\end{array}$ & silt & $\mathrm{C}_{\mathrm{org}}$ & total-S & $\mathrm{SO}_{4}{ }^{2-}$ extr & $\mathrm{DOC}_{\mathrm{extr}}$ & $\mathrm{C}_{\mathrm{hws}}$ & $\mathrm{C}_{1}$ & SRC \\
\hline $\begin{array}{l}\text { Whole } \\
\text { data set }\end{array}$ & 0.512 & 0.346 & 0.341 & 0.294 & 0.021 & -0.056 & 0.132 & 0.700 & -0.213 & 0.040 & 0.171 & -0.024 \\
\hline FFA & 0.626 & 0.441 & 0.428 & 0.370 & 0.007 & -0.176 & -0.196 & 0.347 & 1.426 & 0.811 & 0.364 & -0.185 \\
\hline GKA & 0.503 & 0.345 & 0.259 & 0.208 & -0.206 & -0.080 & 0.750 & 0.670 & -0.789 & -0.133 & 0.170 & 0.039 \\
\hline $\begin{array}{l}\text { non- } \\
\text { sulphidic }\end{array}$ & 0.220 & 0.100 & 0.172 & 0.106 & -0.069 & -0.050 & -1.217 & 0.784 & 0.732 & -1.400 & 0.758 & 1.492 \\
\hline sulphidic & 0.219 & 0.209 & 0.305 & $\begin{array}{c}- \\
0.059\end{array}$ & -0.067 & -0.111 & 1.100 & 0.358 & -2.02 & 0.635 & -0.059 & 0.229 \\
\hline $\begin{array}{l}\mathrm{NO}_{3}^{-}- \\
\text {bearing }\end{array}$ & 0.408 & 0.134 & 0.221 & 0.235 & -0.210 & 0.108 & -1.145 & 0.650 & 1.401 & -0.039 & 0.261 & 0.797 \\
\hline $\mathrm{NO}_{3}{ }^{-}$-free & 0.160 & 0.103 & 0.313 & 0.144 & -0.337 & -0.017 & 0.950 & 0.214 & -2.422 & -0.335 & 0.230 & 0.492 \\
\hline
\end{tabular}



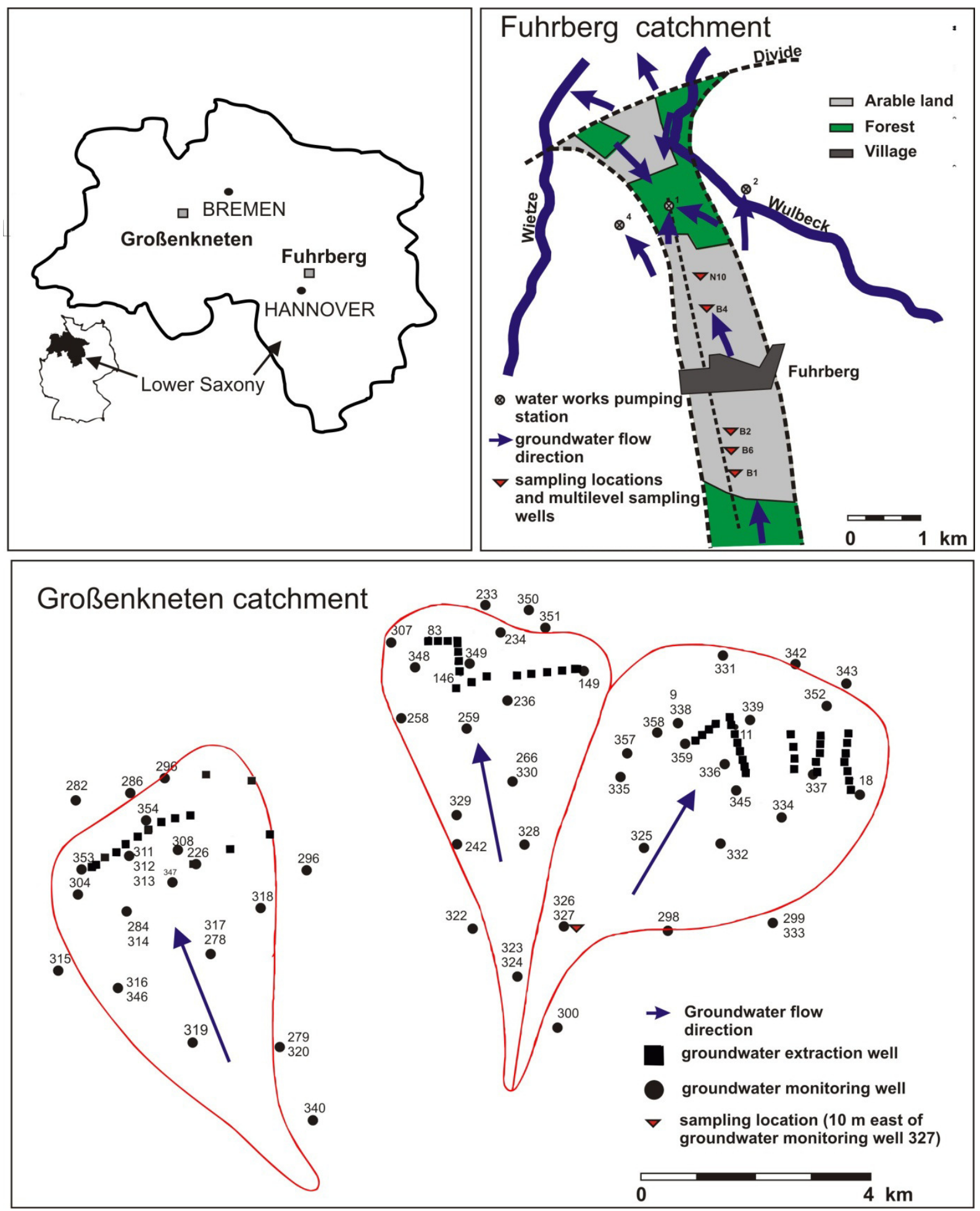

Fig. S1. Sampling locations within the Fuhrberger Feld and Großenkneten catchment in Lower Saxony (Germany). 

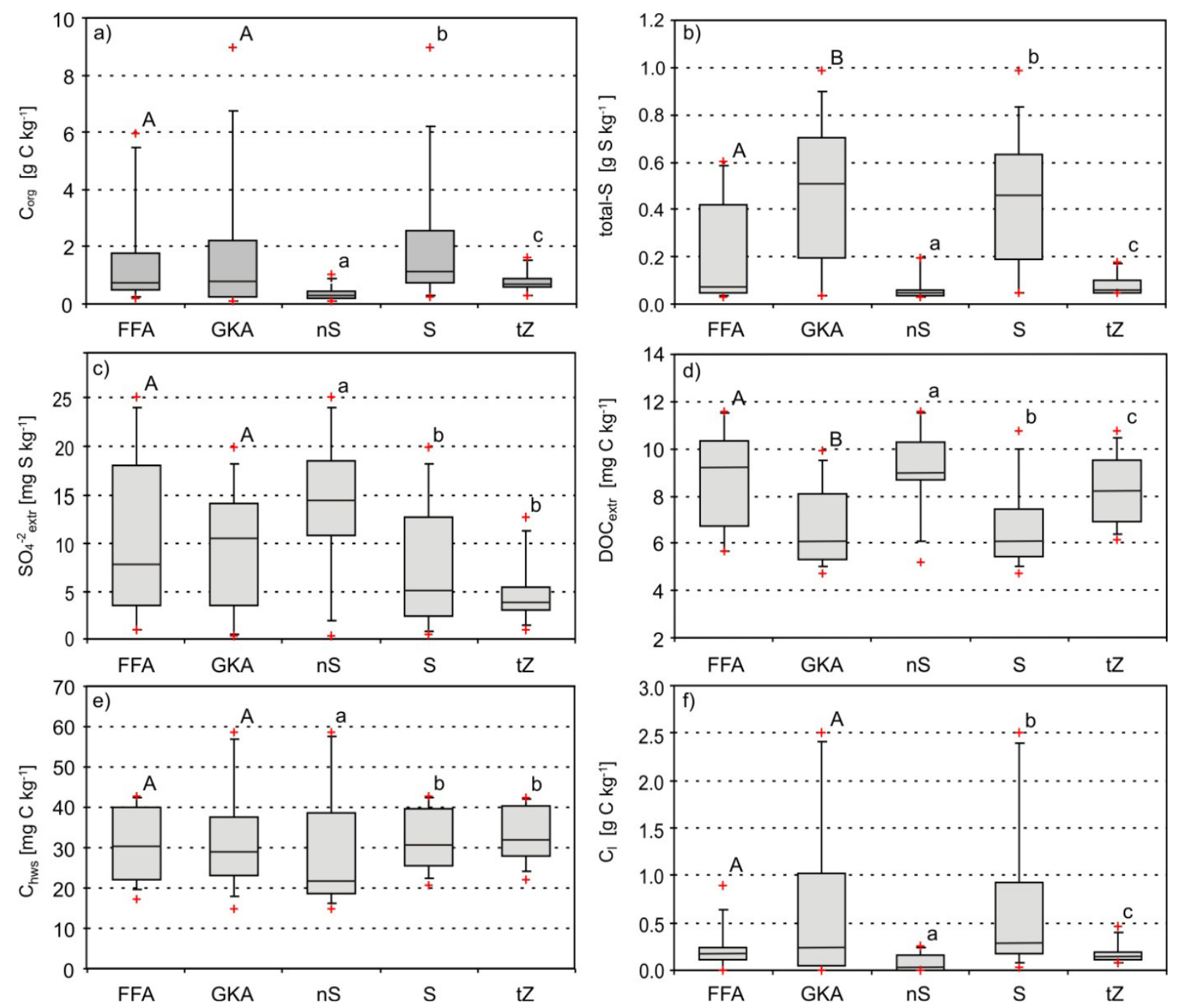

Fig. S2. Distribution of different sediment parameters in the aquifer material from the Fuhrberger Feld aquifer (FFA) and the Großenkneten aquifer (GKA) and in the various established groups of aquifer material: a) organic carbon, b) total sulphur, c) extractable sulphate, d) extractable dissolved organic carbon, e) hot water soluble organic carbon, f) potassium permanganate labile organic carbon. $\mathrm{n} S, \mathrm{~S}$ and $\mathrm{tZ}$ indicate non-sulphidic -, sulphidic and transition zone aquifer material, respectively. Different uppercase letters above the box-plots indicate significant differences between FFA and GKA material, different small letters show significant differences between $n \mathrm{~S}, \mathrm{~S}$ and $\mathrm{tZ}$ (Kruskal-Wallis-Test $(\mathrm{P}<0.05)$ ). 

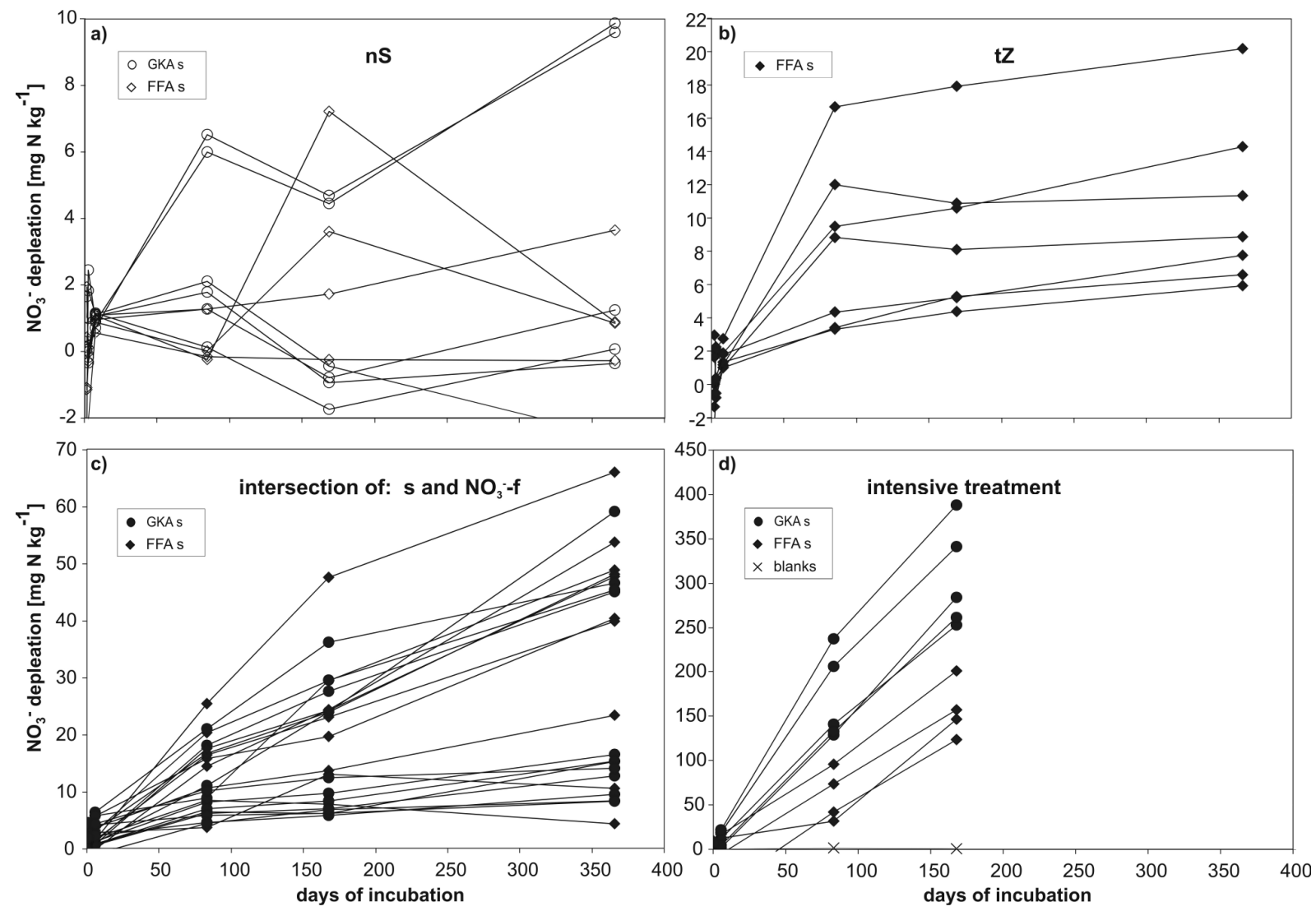

Fig. S3. Measured $\mathrm{NO}_{3}{ }^{-}$consumption during incubations. (The $\mathrm{NO}_{3}{ }^{-}$concentrations at the last sampling date of intensive incubations were not measured.) 


\section{References}

Burgin, A. J., and Hamilton, S. K.: Have we overemphasized the role of denitrification in aquatic ecosystems? A review of nitrate removal pathways, Front. Ecol. Environ., 5, 89-96, 10.1890/1540-9295(2007)5[89:hwotro]2.0.co;2, 2007.

Canfield, D. E., Stewart, F. J., Thamdrup, B., De Brabandere, L., Dalsgaard, T., Delong, E. F., Revsbech, N. P., and Ulloa, O.: A Cryptic Sulfur Cycle in Oxygen-Minimum-Zone Waters off the Chilean Coast, Science, 330, 1375-1378, 10.1126/science.1196889, 2010.

Dalsgaard, T., Thamdrup, B., and Canfield, D. E.: Anaerobic ammonium oxidation (anammox) in the marine environment, Res. Microbiol., 156, 457-464, 10.1016/j.resmic.2005.01.011, 2005.

Eschenbach, W., and Well, R.: Online measurement of denitrification rates in aquifer samples by an approach coupling an automated sampling and calibration unit to a membrane inlet mass spectrometry system, Rapid Commun. Mass Spectrom., 25, 1993-2006, 10.1002/rcm.5066, 2011.

Jetten, M. S. M., Strous, M., van de Pas-Schoonen, K. T., Schalk, J., van Dongen, U., van de Graaf, A. A., Logemann, S., Muyzer, G., van Loosdrecht, M. C. M., and Kuenen, J.

G.: The anaerobic oxidation of ammonium, Fems Microbiol. Rev., 22, 421-437, 10.1111/j.1574-6976.1998.tb00379.x, 1998.

Korom, S. F., Schuh, W. M., Tesfay, T., and Spencer, E. J.: Aquifer denitrification and in situ mesocosms: modeling electron donor contributions and measuring rates, Journal of Hydrology (Amsterdam), 432/433, 112-126, 10.1016/j.jhydrol.2012.02.023, 2012.

Mulvaney, R. L.: DETERMINATION OF N-15-LABELED DINITROGEN AND NITROUS-OXIDE WITH TRIPLE-COLLECTOR MASS SPECTROMETERS, Soil Sci. Soc. Am. J., 48, 690-692, 1984.

Rivett, M. O., Buss, S. R., Morgan, P., Smith, J. W. N., and Bemment, C. D.: Nitrate attenuation in groundwater: A review of biogeochemical controlling processes, Water Res., 42, 4215-4232, 10.1016/j.watres.2008.07.020, 2008.

Schubert, C. J., Durisch-Kaiser, E., Wehrli, B., Thamdrup, B., Lam, P., and Kuypers, M. M. M.: Anaerobic ammonium oxidation in a tropical freshwater system (Lake Tanganyika), Environ. Microbiol., 8, 1857-1863, 10.1111/j.1462-2920.2006.001074.x, 2006.

van de Leemput, I. A., Veraart, A. J., Dakos, V., de Klein, J. J. M., Strous, M., and Scheffer, M.: Predicting microbial nitrogen pathways from basic principles, Environ. Microbiol., 13, 1477-1487, 10.1111/j.1462-2920.2011.02450.x, 2011.

Wall, L. G., Tank, J. L., Royer, T. V., and Bernot, M. J.: Spatial and temporal variability in sediment denitrification within an agriculturally influenced reservoir, Biogeochemistry, 76, 85-111, 10.1007/s10533-005-2199-6, 2005.

Well, R., Becker, K. W., Langel, R., Meyer, B., and Reineking, A.: Continuous flow equilibration for mass spectrometric analysis of dinitrogen emissions, Soil Sci. Soc. Am. J., 62, 906-910, 1998. 